Populations vulnérables

\title{
Faire face ou faire avec. Situations de vulnérabilité et situations de handicap
}

Face it or deal with it. Vulnerabilty situations and handicap situations

Joël Meissonnier

\section{OpenEdition}

1 Journals

Édition électronique

URL : https://journals.openedition.org/popvuln/1249

DOI : 10.4000/popvuln.1249

ISSN : 2650-7684

Éditeur

LIR3S - Laboratoire Interdisciplinaire de Recherche "Sociétés Sensibilités Soin" (UMR 7366 CNRS-uB)

Édition imprimée

Date de publication : 1 décembre 2020

Pagination : 15-37

ISBN : 978-2-918173-28-1

ISSN : 2269-0182

Référence électronique

Joël Meissonnier, "Faire face ou faire avec. Situations de vulnérabilité et situations de handicap », Populations vulnérables [En ligne], 6 | 2020, mis en ligne le 01 décembre 2021, consulté le 26 février 2022. URL : http://journals.openedition.org/popvuln/1249; DOI : https://doi.org/10.4000/popvuln. 1249

Ce document a été généré automatiquement le 26 février 2022.

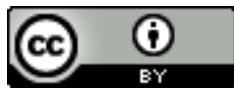

Les contenus de la revue Populations vulnérables sont mis à disposition selon les termes de la Licence Creative Commons Attribution 4.0 International 


\section{Faire face ou faire avec. Situations de vulnérabilité et situations de handicap}

Face it or deal with it. Vulnerabilty situations and handicap situations

Joël Meissonnier

\section{Introduction}

1 Précaires, désaffiliés, exclus, pauvres, handicapés, insulaires et maintenant vulnérables? L'éventail des qualificatifs employés pour désigner des personnes éprouvant des difficultés découlant de leur potentialité à être mobile nuit à l'intelligibilité du problème. Qu'ajoute ou que retire la notion de vulnérabilité dans le contexte d'une analyse des pratiques de mobilité ? Un aperçu de son emploi dans la littérature s'impose. Or une tendance s'esquisse à employer indifféremment - ou de manière assez synonyme - situation de handicap et situation de vulnérabilité en sciences sociales. Cet article plaide en faveur d'une distinction claire entre les deux notions eu égard au caractère récurrent de l'une, impromptu de l'autre. Nous illustrerons l'intérêt de cette différenciation sémantique à partir d'une enquête qualitative consacrée à la mobilité quotidienne des personnes en situation de handicap cognitif et/ou mental réalisée à Amiens et Paris en 2012-2013. Si nous sommes tous victimes lorsqu'un aléa survient au cours d'un déplacement, par définition imprévisible, il peut rendre vulnérables les personnes en situation de handicap plus particulièrement. La distinction sémantique entre situation de handicap et situation de vulnérabilité prend ici tout son sens. Alors qu'il est déjà complexe de faire avec une situation de handicap, faire face à l'aléa qui produit la situation de vulnérabilité relève alors d'un défi supplémentaire, souvent insurmontable. 


\section{Problématique : une question de sémantique pas si anodine}

2 La notion de vulnérabilité doit être utilisée avec précaution, car cette notion ne renvoie pas toujours aux mêmes contenus et n'est pas opérationnalisée de la même manière selon les cas (Alwang et al., 2001, cité par Léon, Pin et Du Roscoät, 2018). Le concept de vulnérabilité apparaît tout d'abord dans les sciences environnementales et de la gestion des risques naturels (Martin, 2013) afin de pointer les inégales capacités des populations à faire face (coping capacities) en situation de catastrophe naturelle. Dans les travaux de Robert d'Ercole et al. (1994), la perspective est élargie à d'autres risques mais demeure une question de protection des populations civiles face à la catastrophe : « la vulnérabilité apparaitt comme la propension d'une société à subir des dommages en cas de manifestation d'un phénomène naturel ou anthropique » ayant un caractère fortuit ou aléatoire. Cette approche fait de la notion de vulnérabilité un concept probabiliste (Chambers, 1989). Elle saisit la relation ou la proximité d'un sujet avec un dommage. Le risque qu'une personne a de subir un dommage (sa vulnérabilité) est le résultat incrémental d'un ensemble de risques distincts mais reliés. Concrètement, il y aurait trois types de risques: le risque d'être exposé à une menace, le risque de matérialisation d'une menace et le risque de manquer des protections nécessaires pour faire face à la menace. (Schröder-Butterfill et Marianti, 2006).

3 C'est en épidémiologie que la notion de vulnérabilité opère un premier glissement sémantique. La dimension exceptionnelle et catastrophique du risque auquel on fait face disparaît, mais la notion conserve sa dimension probabiliste. Une sous-population présente des facteurs de vulnérabilité lorsque la prévalence d'une maladie y est, relativement au reste de la population, statistiquement plus importante. Même si dire que les personnes âgées présentent des facteurs de vulnérabilité aux maladies de la vieillesse est quelque peu tautologique, il est par exemple intéressant de montrer que les personnes âgées présentent une vulnérabilité à l'isolement social (Léon, Pin et $\mathrm{Du}$ Roscoät, 2018) ou au syndrome de Diogène (Bonin, Combe, Fabre, Auguste, Achour et Gonthier, 2018).

4 La sociologie, quant à elle, s'empare de la notion de vulnérabilité à la suite des réserves formulées par Robert Castel à propos de la notion d'exclusion, très populaire à la fin des années 1980. Il envisage d'abord le concept de désaffiliation (Castel, 1990) avant d'en arriver à la notion de vulnérabilité appliquée à des catégories de population. Souvent employée dans des travaux portant sur la pauvreté et ses effets sur les trajectoires des individus et des groupes (Martin, 2013), la notion de vulnérabilité en sociologie tente d'abord d'appréhender des déficits de ressources. La notion perd au passage son optique probabiliste pour alimenter des analyses en termes de capitaux nettement plus déterministes. Le lien avec la catastrophe fortuite s'estompe lui aussi. Comme le soulignent Marie-Aude Corbillé et co-auteurs (2018), cette vulnérabilité sociale, en faisant appel à d'autres notions comme celle de capacité d'adaptation, «nuit à la lisibilité et induit des confusions avec le concept de résilience ».

5 Un dernier glissement sémantique se produit actuellement, lorsque journalistes ou personnalités politiques qualifient de vulnérables des populations entières (Rom, personnes issues de l'immigration, ressortissants d'une ancienne colonie, etc.) pour signifier qu'elles sont victimes de discriminations. Parfois également synonyme de population défavorisée, cet emploi essentialise la différence. Et en dépouillant la notion 
de vulnérabilité de l'aléa et de la probabilité auxquels elle faisait référence, celle-ci devient floue voire stigmatisante.

En sciences humaines et sociales, la notion de vulnérabilité a eu un écho particulier dans le champ du handicap (Collectif, 2017). Au même titre que l'on est passé progressivement d'une approche "physicaliste» (l'infirmité) à une approche normative (la situation de handicap) dès lors que l'on a admis que le manque ne reposait pas simplement sur l'organe et sa fonction, mais également sur la place de l'individu dans la société (Ramadier, 2011), l'approche conceptuelle de type interactionniste envisage la vulnérabilité comme le résultat de différences de capacité relatives, et non plus comme une différence absolue. Dès lors, le concept de vulnérabilité, au même titre que celui de handicap, ne renvoie pas seulement aux populations, mais aussi (et peut-être surtout) aux contextes et aux structures sociales (Fijalkow, 2014). Autrement dit, la notion de vulnérabilité désigne moins un statut qu'un rapport inéquitable. De sorte que l'on devrait davantage parler de situation de vulnérabilité. Hélène Thomas (2008; Collectif, 2017), parle de "situations de risque anormal qu'encourent des groupes humains $\mathrm{du}$ fait de contextes vitaux particulièrement difficiles ou de déficiences individuelles ». Dès lors, la vulnérabilité est toujours relative, résultant d'une comparaison défavorable à une situation «normale» dans un environnement historique et géographique donné. Ce vocable permet de ne pas assener de manière trop définitive un indépassable jugement relatif à une population donnée. La notion ouvre la porte à une expérience sociale, mais aussi à des rapports de force et, enfin, à des (im)possibilités d'agir. Contrairement à la notion d'exclusion, qui peut suggérer l'idée d'une mise à l'écart définitive (Paugam, 1996), elle se conçoit comme un risque urbain qui pèse sur les individus dans la ville globale en tension (Deboulet, 2008) et amène ceux-ci à pouvoir plus ou moins rebondir individuellement ou collectivement (Bouillon et al., 2015) selon des capacités propres et en fonction de ressources disponibles - ou non - dans l'environnement de vie.

7 Concrètement, si l'on se focalise sur les pratiques de mobilité quotidienne, on peut dire qu'à ressources équivalentes, les individus font preuve de capacités relatives. Ainsi en va-t-il de nos (in)capacités à anticiper, à lire une carte ou à supporter l'attente. Lorsque le fonctionnement d'un système technique de transport évolue, certains individus, plus que d'autres, y voient une complexification qui produit chez eux un sentiment d'impuissance ou d'ingérabilité qui fonde leur vulnérabilité en ce qu'ils ont besoin de davantage de repères, d'être mieux informés, d'être accompagnés sans pour autant être infantilisés.

8 Mais alors, peut-on encore établir une différence entre situation de handicap et situation de vulnérabilité? Notre contribution se propose, à l'appui d'une recherche sur les situations de handicap cognitifs et mentaux, de démêler l'une de l'autre. Nous montrerons l'intérêt de réintroduire en sciences sociales la dimension probabiliste que la notion de vulnérabilité avait à l'origine afin de considérer les conséquences d'un risque, de mieux prendre en compte l'aléa ou la perturbation inopinée dans les transports et ainsi de contribuer à l'urgente réflexion qu'une mobilité durable requiert : comment rendre nos réseaux de transports plus résilients? 


\section{Méthodologie}

9 Nous tirons la plupart de nos données du volet qualitatif d'une recherche financée par le programme interministériel de recherche et d'innovation dans les transports terrestres (PREDIT), pilotée par l'IDUP ${ }^{1}$ avec le concours du CEREMA ${ }^{2}$ et de l'IFSTTAR ${ }^{3}$ et réalisée en 2012-2013 à Amiens et Paris. À cette occasion, nous avons accédé à des personnes en lien avec les situations de handicap cognitif ou mental. La recherche ouvre des pistes de préconisations pour rendre l'espace urbain et la voirie accessibles à toutes les formes de handicap, y compris les moins visibles. D'un point de vue méthodologique, nous avons procédé par focus groups (9 à 12 personnes) avec différents publics cohérents :

- personnes dites «naïves» (individus volontaires a priori sans handicap connu, agents de la mairie d'Amiens) ;

- étudiants étrangers (étudiants volontaires de l'université Paris 1, a priori en situation de décalage culturel);

- éducateurs et accompagnants professionnels en SAVS (Service d'accompagnement à la vie sociale) à Paris ;

- parents et proches de personnes en situation de handicap cognitif ou mental (à Amiens).

- représentants d'associations amiénoises de défense des droits des personnes en situation de handicap ;

- jeunes Parisiens atteints d'épilepsies avec pharmaco-dépendances (18-25 ans) ;

- personnes âgées amiénoises (plus de 70 ans).

10 Puis nous avons suivi dans leurs déplacements quotidiens quelques personnes en situation de handicap (deux personnes ayant une trisomie 21, une personne ayant une déficience intellectuelle et une personne dyspraxique ${ }^{4}$ ) à partir de la méthode des parcours commentés filmés (Grosjean et Thibaud, 2001). Il s'agit d'envisager les individus en situation de handicap comme des experts de leurs itinéraires routiniers. La méthode tire parti de cette expertise qui naît conjointement d'une connaissance fine d'un territoire et d'une connaissance vécue et située liée au handicap. Enfin, nous avons proposé des parcours du combattant (Chevrier et Juguet, 2003) aux personnes avec lesquelles une mise en difficulté semblait envisageable. Ce sont des mises en situations factices (par exemple un rendez-vous imaginaire) amenant l'individu à entreprendre un jeu de piste dans l'espace urbain (bien réel) en vue d'atteindre une destination inhabituelle (Meissonnier et Dejoux, 2016).

\section{IV. Être en situation de handicap}

11 Il n'est pas nécessaire de se penser et encore moins d'être reconnu handicapé pour se trouver en situation de handicap. Pour illustrer cette réalité, on donne souvent l'exemple du parent encombré d'une poussette qui éprouve des difficultés à passer un portique. De même, il n'est pas abusif de parler de situation de handicap cognitif lorsque Corinne, rencontrée à Amiens à l'occasion d'un focus group rassemblant des personnes a priori sans handicap dit: "Quand je suis arrivée à Amiens... je ne me déplaçais pas dans Amiens toute seule. C'est mon mari qui m'a fait connaître un peu le nom des rues, mais c'est vrai que j'avais tendance à éviter de sortir, ne pas aller trop loin justement pour ne pas me perdre, à jamais me déplacer toute seule en fin de compte». 
12 L'existence (pour soi, pour autrui ou pour le corps médical) d'un handicap cognitif ou mental n'apparaît souvent qu'avec l'accroissement des occurrences de ces situations où l'autonomie fait défaut. Cumulées, elles rendent palpable le handicap rencontré. Pour Camille (éducatrice) : "L'apprentissage d'un parcours c'est tous les jours ». L'homme auquel elle pense, «même s'il ne va [à son rendez-vous] qu'une fois par semaine », a besoin de s'entraîner "tous les jours » à réaliser l'itinéraire. "C'est comme ça qu'il apprend, parce que, au début on ne faisait [le trajet] qu'une fois par semaine. [Mais] quand il allait à son rendez-vous, il ne mémorisait pas. D'une semaine sur l'autre, il ne mémorisait pas». Les professionnels évoquent l'éternel recommencement que constitue leur travail d'accompagnement aux apprentissages, sous peine d'oubli, d'erreur ou de crise d'angoisse. Proches et parents y participent aussi. Tanguy, père d'une jeune trisomique, confirme : «C'est répéter, c'est le répétitif ». Camille, directrice de SAVS $^{5}$ parle d'une personne avec qui a été réalisé un «livret de parcours » pour mettre en place une routine de mobilité :

Il y a eu la première fois, avec lui. La deuxième on a fait les photos, la troisième fois

[les éducateurs] l'ont accompagné avec les photos dans le repérage, la quatrième ils lui ont fait repérer seul avec les photos, la cinquième fois, avec ou sans les photos comme il voulait, la sixième fois on a dit: «maintenant, c'est tout seul ». [...] Et alors ce qu'on ne lui avait pas dit c'est qu'on le suivait. Il s'est trompé mais oui, il y est arrivé, il y est arrivé à son rendez-vous et la fois d'après, moi j'ai dit, « non on ne le suit plus ", mais par contre il a sur son téléphone portable notre numéro et le numéro de son lieu de rendez-vous. Il sait repérer les touches, enfin on lui met des photos aussi. Il y a les photos des gens, et il appelle s'il se trompe. Sur un trajet d'une demi-heure il a mis $1 \mathrm{~h}$ et quart.

13 On l'oublie parfois mais la personne handicapée n'est pas passive face à la difficulté. Un actif travail de compensation du handicap cognitif ou mental est entrepris par les personnes concernées elles-mêmes. Il rend la situation de handicap surmontable. Ce travail se fait souvent au prix de nombreux essais, d'une intense préparation et d'une longue mise en condition, comme en témoignent ces échanges entre jeunes épileptiques pharmaco-dépendants. Pascal éprouve des difficultés à se rendre à des entretiens d'embauche. Il dit : «Ce que je fais, comme je ne connais pas trop les plans, je pars euh... comment dire, soit à l'avance ou je pars la veille. Comme ça, je fais un repérage ». Liliane, elle aussi, anticipe le moindre déplacement : «Moi, je prépare tout le soir [la veille]. Même les bouteilles d'eau... ». Louise ajoute : "J'écris sur un papier pour bien savoir la station [d'arrivée ou de changement]. Je suis obligée de faire ça sinon je m'y perds avec le plan ». Mylène abonde : «... parce que le matin on ne se rappelle pas». Mohamed complète: "Parce que le matin t'es plus pressé ». Pierre ajoute : «Moi j'oublie souvent de prendre mes tickets de métro alors je suis obligé de retourner chez moi pour prendre mes tickets. Ou sinon la carte Navigo. Mais j'oublie facilement, en fait, de prendre mes affaires ». Mylène poursuit : « Moi avant je ne faisais pas [cette préparation]. C'est pour ça que j'oubliais tout le temps [des choses importantes pour le trajet]. Mais maintenant, si je dois aller ici ou là, je mets tous mes papiers dans mon sac ».

Ce travail de compensation du handicap se poursuit tout au long du déplacement. L'évitement de situations anxiogènes, par exemple, est une préoccupation de tous les instants pour les personnes épileptiques. Il s'agit de minimiser les risques de crise dans l'espace public ou dans les réseaux de transport. Pierre dit : «Dans un métro où il y a du monde, on étouffe, en fait ». Constant confirme qu' « il y a des lignes de métro qui sont blindées ». La tactique de Liliane consiste à ne pas y pénétrer: "Moi j'attends [le 
suivant], je le laisse passer ». Il n'est pas rare qu'elle laisse passer trois métros avant de décider de monter. Mylène explique : «Dès qu'il y a du monde dans le métro j'ai une peur panique, j'évite de monter, je ne monte pas en général. Non, parce que j'ai la tête qui tourne. Des fois, je fais un effort. J'essaye de rentrer. Je me dis bon allez, ça va. Mais souvent je ressors parce que ça me fait... j'ai envie de vomir. [...] C'est pour ça que je suis tout le temps en retard. »

Ainsi, bien souvent, la situation de handicap rend plus compliquée la réalisation d'un déplacement. Elle le ralentit mais ne l'empêche pas complètement. Notamment quand des formes d'accompagnement et de compensation techniques ou tactiques, qu'elles soient motrices ou psychologiques peuvent être imaginées, préparées et mises en œuvre. Toutefois, si les personnes en situation de handicap parviennent à faire avec les difficultés qu'elles rencontrent grâce à un vaste travail d'anticipation en amont, ce travail se limite bien souvent aux situations de transport normales, beaucoup plus difficilement à une situation de transport dégradée ou perturbée. C'est la raison pour laquelle les personnes en situation de handicap sont, plus que d'autres, attachées à leurs routines de mobilité.

\section{Une incontournable routine de mobilité}

Anthony Giddens la place au cœur de sa théorie de la structuration (Giddens, 1987). La routine est à la fois facteur de maintien de la personnalité de l'individu qui se déplace sur des chemins balisés et facteur de stabilité des institutions sociales dont la fonction dépend de leur capacité à se reproduire continuellement. Nos routines de mobilité sont d'indispensables réducteurs de charge mentale. Loin de l'image de prison sans barreau qu'elles véhiculent parfois, les routines sont des pratiques socio-spatiales indispensables et précieuses dans la gestion du temps (Meissonnier et Richer, 2015). Sans elles, le quotidien serait impossible à vivre (Juan, 2015).

Notre enquête nous a mené à Amiens, où nous avons interrogé en focus group des personnes âgées (+ 70 ans). Il est intéressant de constater que la routine, pour ces personnes, est plus complexe que pour d'autres à faire évoluer. Un peu avant l'enquête, les collectivités (EPCI Amiens Métropole) avaient décidé de faire de la gare d'Amiens un pôle d'échange majeur des lignes de bus urbaines et interurbaines et l'entreprise de transport délégataire (KEOLIS opérant sous la marque AMETIS) avait également procédé à un cadencement des lignes de bus. Ces évolutions ont substantiellement modifié la forme du réseau de transports en commun ainsi que les habitudes quotidiennes des usagers.

Lise : Ils vont toujours [nous faire] changer à la gare pour arriver en ville.

Animateur/trice : Et pour vous c'est un problème?

Lise : Ah oui c'est un gros problème.

18 Les logiques de rabattement sur pôles d'échanges et de cadencement des bus, paraissent difficiles à intégrer et à accepter :

Noémie : Quand je suis arrivée à Amiens, il y avait un système de bus qui passait dans le centre-ville.

Violette : Maintenant il y a beaucoup de lignes de bus qui passent par la gare, qui contournent les boulevards qui vont à la maison de la culture, mais le centre-ville est complètement ignoré. [Passer par la rue piétonne] des trois cailloux, c'était beaucoup plus facile avant. On perd du temps, on monte, on descend du bus, on remonte dans un autre bus. 
Noémie : Si on a un rendez-vous, moi je ne sais pas... un médecin ou aller quelque part. S'il faut prendre deux bus et s'il faut finir un peu à pied...

Violette : [Il y a] ce qu'ils appellent leur fameux cadencement. Mais qui n'existe pas parce qu'il n'y a pas suffisamment de bus. Quand vous avez un changement et que vous devez attendre 20 minutes c'est inutile d'attendre [la correspondance].

D'un point de vue cognitif, la rupture des routines est perturbante pour ces personnes âgées. Elles vivent comme un poids l'effort supplémentaire que leur demande la rupture de charge dans un pôle d'échanges de la ville avant d'atteindre l'hyper-centre piétonnier. Mais ce n'est rien à côté de l'impermanence. En effet, une mesure à destination des personnes à mobilité réduite a été mise en place pour compenser la piétonisation de l'hyper-centre d'Amiens. Elle s'est concrétisée par la mise en place d'une navette électrique innovante. Mais cette navette a eu tant de déboires techniques que le service a été interrompu quelques mois. Puis il a repris, avec d'autres véhicules et un autre design. Avant d'être finalement définitivement arrêté.

Denise : À un moment, il y avait des petits bus qui allaient, qui parcouraient la ville.

Noémie : Des voiturettes, des navettes

Denise : Ils les ont supprimées !

Animateur/trice : Pour vous, c'était mieux les navettes?

Denise : Bah c'est moins haut et puis...

Lise : C'est plus praticable.

Noémie : Ça permettait d'avoir le centre-ville [à portée].

Cette impermanence engendre des changements de comportement très concrets pour les personnes âgées dont la recherche d'alternatives est coûteuse d'un point de vue cognitif. Elle a largement contribué à ce que Denise ne circule plus dans l'espace public.

Denise : Au début il y avait 10 [voiturettes] après il n'y en avait plus que 5, et puis après, plus que 3. Et au bout de [quelques mois] il n'y avait plus de batterie, donc ils ferment. Moi je circulais beaucoup avec ça. Mais maintenant je me fais livrer [les courses].

21 L'impermanence suscite de la frustration chez ces personnes âgées qui avaient pris goût à un nouveau mode de transport adapté à leurs besoins.

Noémie : Ils ne prenaient que des personnes âgées en fait, et ça aidait bien. Pour aller à la mairie, de la gare... on a du mal ; ça fait loin. Et comme les rues sont piétonnes...

Denise : C'était bien ça. On les appelait par téléphone et ils venaient à un endroit précis.

22 Un même attachement à la routine de mobilité a pu être observé parmi les jeunes épileptiques interrogés, avec une personne ayant une déficience intellectuelle, avec deux personnes trisomiques ainsi qu'avec une personne ayant une dyspraxie et que nous avons accompagnées lors de parcours commentés. La routine stabilisée présente un immense intérêt pour de nombreuses personnes en situation de handicap cognitif ou mental : elle évite d'avoir à prendre de trop nombreuses décisions. Elle est une garantie sur la destination puisque les choses se déroulent selon un script maitrisé.

Les jeunes épileptiques que nous avons fait participer au focus group sont venus à l'hôpital où ils ont l'habitude de se rendre. L'itinéraire est connu et routinier. Mylène est arrivée en retard parce qu'elle est partie de chez elle un peu tard. Elle explique qu'elle avait le choix : "soit le bus soit le métro » pour venir. Mylène sait que «le bus, c'est plus long que le métro ». Mais elle a préféré prendre le bus car c'est le mode de transport qu'elle utilise habituellement. Prendre le métro aurait constitué, le cas échéant, un événement perturbateur générateur d'angoisse: "[Si j'étais arrivée] en 
métro, il [aurait fallu] longer l'hôpital... on voit plein de malades... Je n'aime pas les hôpitaux ».

L'enquête montre un fort attachement aux routines des personnes en situation de handicap cognitif et mental. Quand il s'agit de parcourir un itinéraire moins familier, Mohamed (jeune épileptique) est adepte d'une logique heuristique. Il trouve son chemin par une succession d'essais-erreurs. "Oui, [je cherche] jusqu'à ce que je trouve ", dit-il. Ainsi, au sein d'un pôle d'échanges, il essaye tous les couloirs jusqu'à trouver le bon. À un carrefour, il s'engage dans toutes les rues jusqu'à trouver la bonne. Constant (autre jeune épileptique), au contraire, préfère suivre une logique procédurale algorithmique. Il suit à la lettre les consignes d'un itinéraire donné par Google Maps : "Sur Google ils mettent : prenez à droite, prenez à gauche ». Il imprime la liste des actions à suivre dans l'ordre chronologique. Habituellement, on arbitre entre l'une ou l'autre de ces logiques selon leur pertinence étant donné les informations dont on dispose. Mais Mohamed et Constant éprouvent des difficultés à passer de l'une à l'autre, à choisir la plus appropriée selon les circonstances. Ces formes de rigidités cognitives les amènent à éviter les itinéraires les moins familiers et à toujours préférer les plus routiniers.

\section{C'est l'aléa qui place les personnes handicapées en situation de vulnérabilité}

Le plus souvent, les personnes parviennent, par diverses mesures compensatoires anticipées, à s'accommoder de leurs handicaps au quotidien. L'aléa, en revanche, prend de court tous les usagers et place en situation de vulnérabilité les personnes déjà en situation de handicap plus particulièrement. Des témoignages nous permettent de décrire et de mieux saisir ce basculement vers la situation de vulnérabilité lorsque survient une crise inopinée. Florent, jeune épileptique, explique comment est survenue l'une de ses crises :

Florent: Dans la descente de l'escalier mécanique, j'ai dégringolé. Quand j'arrive en bas... il y a un homme et une femme. La femme a voulu m'aider et son mec euh... [...], il sort à sa femme : «ne l'aide pas ; peut-être que c'est un drogué ; pourquoi tu t'en approches?»

Animateur/trice : Vous êtes conscients durant vos crises et vous vous souvenez de tout?

Florent: Moi oui.

Mohamed : En fait, c'est le coup de surprise.

Pascal: Moi, ça m'est arrivé un jour. D'ailleurs je crois que c'était la première, c'était la plus violente. Les convulsions... enfin bref. Au moment où j'allais commencer à avoir ces symptômes, une personne est venue [vers moi], j'ai commencé à dire « aide-moi, aide-moi, aide-moi, il est en train de se passer quelque chose » et après je suis tombé et je ne me suis plus rappelé de rien.

Florent: Moi, si je sens une grosse crise. Je prends le mur, je m'y agrippe jusqu'à ce que je descende au sol.

Les situations de crise évoquées ci-dessus constituent une exception. Le handicap, en lui-même, survient rarement par surprise et la crise d'épilepsie est un cas singulier de ce point de vue dans la mesure où l'aléa est (partiellement au moins) endogène. Le plus souvent, celui-ci est exogène (un incident ou un accident, une grève sans préavis, une inondation, des travaux sur une ligne de transport, etc.). Mais ces moments de crises 
impromptus peuvent être tout aussi incapacitants pour les personnes en situation de handicap cognitif ou mental.

L'événement impromptu constitue l'une des principales sources d'angoisse pour Pascal, jeune épileptique, car il réclame une activité cognitive plus intense, une attention redoublée nécessitant une prise de décision. «S'il y a des travaux » qui occasionnent une déviation de la ligne, Pascal se demande s'il « doit s'arrêter avant pour prendre un autre bus». Et cette perspective n'est pas faite pour le rassurer: «c'est plus compliqué ». Lorsque survient l'événement impromptu et imprévisible, Mylène doit s'en remettre à une autre personne. Elle se souvient: "À Châtelet, je savais plus ou moins mon trajet, même si de temps en temps j'étais un peu perdue. Voilà, moi j'étais habituée, je savais que la ligne D fallait la prendre... je sais plus... du côté Z [sur le repère $Z$ ? quai $Z$ ?]». Mais quand en situation d'incident ou de travaux "on nous demande d'aller, à la dernière minute, prendre le bus, le 7 à côté pour aller à gare de Lyon bah... une fois j'étais obligée de suivre une personne. J'ai dit : «je vous suis ». Bah oui parce que je ne savais pas. On devait aller au même endroit, on s'arrêtait au même endroit... je lui ai demandé, je lui ai dit que je la suivais et je l'ai suivie ». Pour Mylène, la question angoissante est de savoir si elle va trouver une personne de confiance qu'elle parviendra à suivre lorsque survient l'imprévu.

L'événement impromptu est craint d'une part parce qu'il vient remettre en question une habitude ancrée, nous l'avons souligné, d'autre part parce que, pour les personnes interrogées, il contrarie l'enjeu associé à la destination à atteindre. Pascal précise : "J'avais rendez-vous". Malheureusement, ce jour-là, les choses ne se sont pas déroulées comme prévu : « ce bus-là, il est parti de l'autre côté, côté Mont-Mesly et, en fait, c'est pas le côté qu'il fallait qu'il prenne». L'événement est en lien avec les «travaux» de prolongation de la ligne 8 du métro «à Pointe du Lac» [Créteil]. Et concrètement, Pascal n'est pas parvenu à l'heure à son rendez-vous.

Comme le mentionnent Stéphane Juguet et Stéphane Chevrier (2003) dans leur analyse de la désorientation, en situation de crise, les voyageurs sont tous "soumis à un syndrome de surcharge cognitive. Pour ajuster leurs plans d'action, ils doivent déchiffrer des informations contextuelles qui s'inscrivent dans l'environnement immédiat, effectuer, si nécessaire, un calcul en temps réel, interpréter des signes sans pouvoir mobiliser des supports cognitifs qui les auraient allégés ». Pour atteindre leur nouvelle destination, "ils doivent s'appuyer sur l'environnement immédiat et mobiliser des savoir-faire qui, en situation de routine, sont inutiles». Les auteurs remarquent cependant que « face à cette difficulté dans le traitement de l'information, des disparités prononcées apparaissent entre profils " d'individus. Les personnes en situation de handicap deviennent vulnérables en situation d'exception (grèves, travaux, incidents, pannes, etc.).

30 Parmi les jeunes épileptiques interrogés, certains éprouvent de manière récurrente un sentiment d'angoisse. Si la marche a pu être évoquée comme rassérénante par certaines personnes (être à l'air libre, mobilité à échelle humaine, arbitrages qui se succèdent à un rythme apaisé, etc.), ce point de vue fait débat dès qu'on aborde la question de l'insécurité. Et certains soulignent toute la vulnérabilité du piéton en ville, qu'il soit dans la rue ou dans un couloir du métro.

Pierre : Dans la rue je me retourne souvent en fait. [...] Pour [vérifier que] personne ne m'suit. 
Florent : [rire] Pourquoi t'as une mallette?

Pierre : C'est une habitude... C'est comme dans le métro en fait.

Animateur/trice : La peur...?

Pierre : La peur ouais.

Animateur/trice : ... que quelqu'un te suive?

Pierre : Ouais c'est ça.

Animateur/trice : Et t'as peur au fond de quoi, qu'on te fasse du mal ?

Pierre : Agressé, voilà.

Animateur/trice : Ça t'est déjà arrivé?

Pierre : Je me suis fait déjà agresser une fois. C'était dans la rue ouais. [C'est] pour ça [que] maintenant je surveille toujours...

Animateur/trice : ... derrière toi ?

Pierre : Ouais derrière moi.

Animateur/trice : Et donc marcher à pied... vous évitez ?

Pierre : [Autant que je peux] je reste chez moi. Non, mais moi je ne sors jamais le soir comme j'ai peur du noir. Je reste enfermé chez moi avec les lumières allumées.

Constant (épileptique) : Il y a des quartiers où je fais attention et dans le métro je fais encore plus attention.

Animateur/trice : Pourquoi encore plus?

Constant: Parce que... je me suis déjà fait agresser une fois dans le métro. Je fais attention à ne pas sortir mon portefeuille quand je prends mon ticket, à sortir [juste] mon ticket de métro ou bien chez moi, ou bien en bas de chez moi, enfin, j'essaye si possible de pas avoir besoin de sortir mon portefeuille dans le métro ou alors, si je dois l'acheter, si c'est un jour où je n'ai plus de ticket, je prévois chez moi de sortir ma carte pour acheter des tickets et je la mets dans une poche et après, je sors juste ma carte pour acheter des tickets. Je ne veux plus sortir mon portefeuille parce que je sais que... je sais que ça serait trop risqué.

31 Cette méfiance fait écho au parcours commenté d'Étienne, trisomique en grande difficulté de communication. Il souligne à plusieurs reprises la crainte des brimades qu'on a pu lui faire subir dans les transports en commun. La situation de crise dépasse donc le simple dysfonctionnement technique. Elle est parfois produite par l'environnement social. Au-delà d'une plus grande vulnérabilité face à des risques statistiquement fondés - par exemple la probabilité plus grande de ne pas savoir surmonter un dysfonctionnement momentané du réseau de transport - la situation de handicap accroît la vulnérabilité des personnes face aux jugements sociaux dont ils font l'objet et aux procès stigmatisants dont ils sont parfois victimes en de telles circonstances.

\section{Quand le grain de sable se fait montagne}

Noémie et Marguerite sont deux personnes âgées rencontrées lors d'un focus group à Amiens. Attardons-nous sur leur discussion :

Noémie : Pour arriver à prendre les transports en commun à Amiens, il faut... 
Marguerite : Il faut faire attention aux arrêts.

Noémie : ... aux arrêts, à tout.

Animateur/trice : Il faut être vigilant?

Noémie : Oui, voilà.

Marguerite : Et il faut surtout... connaître tous les arrêts d'une ligne.

Animateur/trice: Donc ça veut dire que quand il y a un changement sur la ligne vous réapprenez les différents arrêts ?

Noémie : Ah bah il faut...

Marguerite : Après, faut voir les correspondances. C'est une gymnastique.

Noémie : Oui, ça s'travaille. Et en plus ici c'est compliqué parce que quand la société des transports change les horaires...

Marguerite : ... ou change les lignes...

Noémie : ils n'préviennent pas !

Lise : Ça fait comme les grèves, hein. On n'en sait rien... celui qui prend pas le journal [ne sait pas].

La moindre évolution du réseau de transport en commun réclame, de la part de ces personnes âgées, de consulter leurs sources d'information habituelles (ici le journal local). Que l'on qualifie - ou non - (d'un point de vue médical) la rigidité de leurs habitudes de handicap cognitif, on comprend à quel point le moindre changement produit une angoisse chez ces femmes. Un grain de sable suffit, dans l'enchaînement des modes de transports interconnectés, pour faire basculer certaines personnes en situation de handicap dans une situation de vulnérabilité. D'après Camille, éducatrice rencontrée lors d'un focus group rassemblant des professionnels du handicap, les personnes en situation de handicap mental peuvent perdre tout sens de l'orientation, si elles choisissent mal leurs points de repère. Pour les repères spatiaux l'important est leur permanence, la garantie de les trouver, toujours disponibles pour "faire sens", toujours situés aux mêmes endroits. "Dans le métro, on ne peut pas se fier aux affiches qui changent tout le temps et il y a des stations qui se ressemblent quand même énormément ». Les éducateurs amènent donc les patients pris en charge en SAVS à prendre pour repère « un symbole qui est pérenne » et particulièrement discriminant.

Constant, jeune épileptique, décrit comment un défaut de continuité dans le système de numérotation des immeubles dans la rue - défaut au demeurant parfaitement anodin produit chez lui une profonde perturbation :

Si tu as un rendez-vous à un endroit. Tu as ton numéro d'adresse. [Dans la rue], il y a des bâtiments. Quelques fois, tout d'un coup, y'en a un, il a son numéro [et celui $\mathrm{d}^{\prime}$ ] après on ne sait pas pourquoi, il n'a plus de numéro, [et le suivant] il recommence à avoir un numéro. On ne sait pas trop pourquoi. Tu te dis:bon regardons je marche dans un sens, voyons voir le numéro suivant. Mais ah ! Est-ce que j'vais dans le bon sens des numéros ou dans le mauvais sens des numéros? [...] Si t'es parti dans le mauvais sens - tu étais au 60 et tu passes au 64 - tu te dis : mince j'ai fait tout ce chemin et va falloir [revenir], j'ai pas trouvé le $n^{\circ} 62$ entre-temps ça fait beaucoup de chemin que je viens de me faire pour [rien]. c'est des immeubles [d'habitation] il y a le numéro mais quand c'est un commerce ou un 
restaurant, ils ne mettent pas leur numéro ». Florent ajoute : «Ou ils le mettent en haut ». Mohamed interprète : «Tu l'vois pas ». Et Constant de conclure : « Moi j'trouve ça pénible, les commerces ».

En situation perturbée, la mobilité est vécue différemment d'un individu à l'autre. Les personnes déjà en situation de handicap cognitif peuvent être subitement placées en situation de vulnérabilité au moindre grain de sable. Même si une difficulté se pose à tous, elle se pose de façon plus aiguë aux personnes en situation de handicap.

La mobilité contemporaine se traduit par une multiplication des interactions hommesobjets: le corps-à-corps avec les véhicules (Warnier, 1999), l'interaction avec la multitude des affordances (Gibson, 1977), avec des attracteurs cognitifs et sources d'informations distribuées dans l'environnement (Denis, Pontille, 2010) ainsi que des interactions particulières avec les objets nomades que l'on véhicule avec soi et qui aident à s'orienter, à communiquer ou à supporter l'attente (Chevrier et Juguet, 2003). Or chacune de ces interactions constitue autant de risques de dysfonctionnement ou d'aléas potentiels supplémentaires. Malgré l'existence d'applications numériques pour assister les usagers, les réseaux de transport réclament de leur part toujours plus de capacités d'abstraction, de compétences d'anticipation ou de facultés de déduction. Les personnes en situation de handicap cognitif, ayant besoin de mettre en place des routines nécessitant le moins d'arbitrages possible, sont les premières victimes de ces évolutions majeures.

\section{Conclusion}

Nos résultats d'enquête plaident en faveur d'une différenciation claire entre situation de handicap et situation de vulnérabilité car un risque aléatoire ou un handicap impactent différemment les personnes dans leur mobilité et les exposent à des conséquences peu comparables. Lorsque le handicap est connu et que ses conséquences peuvent être anticipées et gérées, la personne en situation de handicap cognitif ou mental, souvent, continue à se déplacer et parvient à surmonter son handicap. Différencier l'une et l'autre notion, c'est admettre et reconnaître les tactiques résilientes que les personnes en situation de handicap mettent en œuvre quotidiennement pour faire avec. La situation de handicap rend plus compliquée la réalisation d'un déplacement mais souvent, ne l'empêche pas. Un risque ou un aléa nous rend tous vulnérables mais cette vulnérabilité affecte en priorité les personnes en situation de handicap. Face à la matérialisation du risque, elles ne disposent pas de toutes les ressources pour y faire face. Les conséquences peuvent donc en être aggravées. Pour les personnes en situation de handicap cognitif ou mental plus particulièrement, la routine est rassurante, le changement est suspect et l'impermanence désastreuse. De leur point de vue, l'événement impromptu est générateur d'une angoisse déstabilisante, souvent paralysante. Alors qu'il peut être possible de faire avec une situation de handicap, il est souvent plus compliqué de faire face à l'aléa qui caractérise une situation de vulnérabilité. Il nous semble donc important de résister à la tendance à l'utilisation de l'une pour l'autre des notions de situation de handicap et de situation de vulnérabilité en sciences sociales. 


\section{BIBLIOGRAPHIE}

Alwang J., Siegel P. B. et Jorgensen S. L. (2001), « Vulnerability as Viewed from Different Disciplines ", International Symposium Sustaining Food Security and Managing Natural Resources in Southeast Asia - Challenges for the 21st Century, Chiang Mai, Thailand.

Bonin M., Combe L., Fabre F. et al. (2018), « Une vulnérabilité cachée et méconnue du sujet âgé : le syndrome de Diogène », Populations vulnérables, $\mathrm{n}^{\circ} 3$.

Bouillon F., Deboulet A., Dietrich-Ragon P. et al. (2015), « Les vulnérabilités résidentielles en questions », Métropolitiques, disponible sur http://www.metropolitiques.eu/Les-vulnerabilitesresidentielles.html.

Castel R. (1990), « Le roman de la désaffiliation. À propos de Tristan et Iseult », Le Débat, $\mathrm{n}^{\circ}$ 61, p. $152-164$

Chambers R. (1989), « Vulnerability, coping and policy », IDS Bulletin, Institute of Development Studies, University of Sussex, vol. 20, $\mathrm{n}^{\circ}$ 2, p. 1-7.

Chevrier S. et Juguet S. (2003), Arrêt demandé, Paris - Rennes, Enigmatek-LARES.

Collectif [Comité de rédaction] (2017), « Une nouvelle collection : "Populations vulnérables" ", Populations vulnérables, $\mathrm{n}^{\circ} 1$.

Orbille M-A., Jouannic G., Leroy-Dutilleul I. et al. (2018), « Appropriation des notions de vulnérabilité et de résilience : enseignements de 3 territoires inondables », La Houille Blanche, $\mathrm{n}^{\circ} 4$, p. 13, DOI : $10.1051 / \mathrm{lhb} / 2018037$.

Deboulet A. (2008), Restructurations urbaines et construction des compétences. Vulnérabilités et implications des citadins dans les villes internationales, université Lyon II, habilitation à diriger des recherches en sociologie.

Denis J. et Pontille D. (2010), Petite sociologie de la signalétique. Les coulisses des panneaux du métro, Paris, Presses des Mines.

D’Ercole R., Thouret J.-C., Dollfus O. et Asté J.-P. (1994), « Les vulnérabilités des sociétés et des espaces urbanisés : concepts, typologie, modes d'analyse. Revue de géographie alpine, t. $82, \mathrm{n}^{\circ} 4$, p. 87-96.

Fijalkow Y. (2014), « Le logement indigne : une nouvelle norme de l'action publique ?», Informations sociales, $\mathrm{n}^{\circ} 184$, p. 20-32.

Gibson J. J. (1977), « The Theory of Affordances », in Robert S. et Bransford J. (dir.), Perceiving, Acting, and Knowing, Hillsdale, NJ. Lawrence Erlbaum Associates, p. 67-82.

Giddens A. (1987), La constitution de la société, Paris, PUF.

Grosjean M. et Thibaud J-P. (dir.) (2001), L'espace urbain en méthodes, Marseille, Édition Parenthèses.

Juan S. (2015), « Le concept de routine dans la socio-anthropologie de la vie quotidienne », Espace populations sociétés, 2015/1-2, mis en ligne le 1 juillet 2015, disponible sur http:// journals.openedition.org/eps/5935 ; DOI : 10.4000/eps.5935.

Léon C., Pin S. et Du Roscoät E. (2018) «Étude de trois situations de vulnérabilité chez les personnes âgées de 55 à 85 ans en France », Populations vulnérables, n 3. 
Martin C., (2013), « Penser la vulnérabilité : les apports de Robert Castel », Alter. European Journal of Disability Research, Paris, Elsevier Masson, p. 293-298.

Meissonnier J. et Dejoux V. (2016), « The Commented Walk Method as a Way of Highlighting Precise Daily Mobility Difficulties. A Case Study Focusing on Cognitive or Mental Diseases ", Transport Research Arena (TRA2016) Procedia, vol. 14, p. 4403-4409.

Meissonnier J. et Richer C. (2015), « Métro - boulot - dodo : quoi de neuf dans nos routines de mobilité ? », Espace populations sociétés, 2015/1-2, disponible sur http://journals.openedition.org/ eps/5931.

Paugam S. (1996), L'exclusion. L'état des savoirs, Paris, La Découverte.

Ramadier T. (2011), «L'accessibilité socio-cognitive », Actes du 11 eolloque Mobilités spatiales et fluidité sociale, Grenoble.

Schröder-Butterfill E. et Marianti R. (2006), « A framework for understanding old-age vulnerabilities ", Ageing and Society, n²6/1, p. 9-35.

Thomas H. (2008), « Vulnérabilité, fragilité, précarité, résilience, etc. De l'usage et de la traduction de notions éponges en sciences de l'homme et de la vie », Recueil Alexandries, $\mathrm{n}^{\circ} 13$, disponible sur http://www.reseau-terra.eu/article697.html.

Warnier J-P. (1999), Construire la culture matérielle. L'homme qui pensait avec ses doigts, Paris, PUF.

\section{NOTES}

1. IDUP : Institut de démographie de l'Université Paris 1.

2. CEREMA: Centre d'études et d'expertise sur les risques, l'environnement, la mobilité et l'aménagement.

3. IFSTTAR: Institut français des sciences et technologies des transports, de l'aménagement et des réseaux.

4. La dyspraxie est un trouble neurologique chronique qui apparaît dès l'enfance. La maladie se caractérise par une affection de la planification des mouvements et la coordination en raison d'une altération de la communication entre le cerveau et le corps. Malgré l'absence de toute paralysie ou parésie des muscles impliqués dans le mouvement, le sujet doit contrôler volontairement chacun de ses gestes, ce qui est très coûteux en attention et rend la coordination des mouvements de la vie courante extrêmement difficiles. (Source Wikipédia).

5. Service d'accompagnement à la vie sociale.

\section{RÉSUMÉS}

Nous sommes tous victime lorsqu'un aléa survient au cours d'un déplacement (grève, panne, situation climatique exceptionnelle, etc.), par définition imprévisible. Mais il peut rendre vulnérables les personnes en situation de handicap cognitif et/ou mental plus particulièrement. La distinction sémantique entre situation de handicap et situation de vulnérabilité doit ici garder tout son sens. Et la tendance à employer indifféremment l'une et l'autre notion en sciences 
humaines et sociales, mérite d'être dénoncée. Différencier l'une et l'autre notion, c'est admettre et reconnaître les tactiques résilientes que les personnes en situation de handicap mettent en œuvre quotidiennement pour compenser le handicap. Ne pas le faire, c'est nier leurs efforts pour parvenir à se déplacer de façon autonome en situation ordinaire non-perturbée. L'intérêt de cette distinction sémantique est illustré par les résultats d'une enquête qualitative menée en 2012-2013 à Amiens et Paris. Alors qu'il est déjà complexe de faire avec une situation de handicap, faire face à l'aléa qui produit la situation de vulnérabilité relève alors d'un défi supplémentaire, beaucoup moins surmontable.

We are all victims when a hazard occurs during a daily trip (strike, breakdown, exceptional climate...), intrinsically unpredictable. But it can make people with cognitive and / or mental disabilities more vulnerable. The semantic distinction between a situation of disability and a situation of vulnerability must here keep its meaning. And the tendency to use synonymously these two notions in the human and social sciences, have to be denounced. We admit and recognize the resilient tactics that people with disabilities implement on a daily basis to compensate for disability thanks to these two different concepts. Synonymously employed, they deny their efforts to move autonomously in an ordinary, undisturbed situation. The interest of this semantic distinction is illustrated by the results of a qualitative survey conducted in 2012-13 in Amiens and Paris. While it is already complex to deal with a situation of disability, facing the hazard that produces the situation of vulnerability is an additional challenge, much less overcomable.

\section{INDEX}

Mots-clés : comportement de mobilité, situation de handicap, situation de vulnérabilité, handicaps cognitif et/ou mental

Keywords : mobility behaviours, situation of disability, vulnerability context, people with cognitive and/or mental deficiencies

\section{AUTEUR}

\section{JOËL MEISSONNIER}

CEREMA - Équipe de recherche ESPRIM 